\section{References}

1. Butler J, Stankewicz MA, Wu J, Chomsky DB, Howser RL, Khadim G, et al Pre-transplant reversible pulmonary hypertension predicts higher risk mortality after cardiac transplantation. J Heart Lung Transplant. 2005;24:170-7.

2. Humbert M, Morrell NW, Archer SL, Stenmark KR, MacLean MR, Lang IM, et al. Cellular and molecular pathobiology of pulmonary arterial hypertension. J Am Coll Cardiol. 2004;43(12 Suppl S):13S-24S.

3. Haddad H, Elabbassi W, Moustafa S, Davies R, Mesana T, Hendry P, et al. Left ventricular assist devices as bridge to heart transplantation in congestive heart failure with pulmonary hypertension. ASAIO J. 2005; 51:456-60.

4. Ito T, Ozawa K, Shimada K. Current drug targets and future therapy of pulmonary arterial hypertension. Curr Med Chem. 2007;14: 719-33.

5. Mogollon MV, Lage E, Cabezon S, Hinojosa R, Ballesteros S, Aranda A, et al. Combination therapy with sildenafil and bosentan reverts severe pulmonary hypertension and allows heart transplantation: case report. Transplant Proc. 2006;38:2522-3.

\title{
Total resection and complete reconstruction of the tricuspid valve in acute infective endocarditis
}

\author{
Igor E. Konstantinov, MD, PhD, Perth, Australia
}

1 cute infective endocarditis of the tricuspid valve (TV) in noncompliant intravenous drug abusers presents a difficult problem. Progressive sepsis despite appropriate antibiotics necessitates surgical intervention. These noncompliant patients, however, often leave the hospital against medical advice. The risk of prosthetic endocarditis in this group of patients is very high. Complete resection of the valve without replacement, although feasible, can result in progressive right-sided heart failure. Reconstruction of the TV might be an alternative option.

\section{Clinical Summary}

A 33-year-old man with a long history of intravenous drug abuse was admitted with fever and chills. He previously underwent drainage of an infected left hip joint, as well as multiple bilateral groin abscesses at the site of drug injection. Multiple blood cultures demonstrated Staphylococcus aureus. Despite appropriate antibiotic coverage, uncontrolled sepsis developed. Echocardiographic analysis demonstrated large multiple vegetations of the TV (Figure 1, A) and an abscess extending into the free wall of the right ventricle. This infective process involved all 3 leaflets, causing TV stenosis (Figure 1,C).

The patient underwent urgent surgical intervention. Total resection of the TV and the entire subvalvular apparatus was performed. The right ventricular abscess was drained. The entire operation was performed on the beating heart with cardiopulmonary bypass. The

From the Department of Cardiothoracic Surgery, Sir Charles Gairdner Hospital, Perth, Australia.

Received for publication Dec 20, 2007; accepted for publication Dec 24, 2007.

Address for reprints: Igor E. Konstantinov, MD, PhD, Department of Cardiothoracic Surgery, Sir Charles Gairdner Hospital, G Block, Hospital Avenue, Perth, WA 6009 Australia (E-mail: konstantinov.igor@alumni.mayo.edu).

J Thorac Cardiovasc Surg 2008;136:531-2

$0022-5223 / \$ 34.00$

Copyright (C) 2008 by The American Association for Thoracic Surgery doi:10.1016/j.jtcvs.2007.12.061
TV was reconstructed with autologous pericardium soaked in rifampicin (Figure 1, $B-D$ and $E$ ) and polytetrafluoroethylene* cords. Apart from 4-0 polytetrafluoroethylene cords and 4-0 Prolene sutures, no artificial material was used. After complete resection of all infected tissues, a distance from the papillary muscles or right ventricular wall was measured by using a Silastic tube at approximately two thirds of the distance to the TV annulus (Figure 2, A). A polytetrafluoroethylene suture was then placed through the free edge of the reconstructed leaflet, right ventricular myocardium, and pericardial pledget and introduced into the previously sized tube (Figure 2, B). The polytetrafluoroethylene cord was then tightened with the tube in place to prevent incidental shortening of the cord while tightening the knots (Figure 2, $C$ ). The tube was then removed, and the reconstructed leaflet was retracted (Figure 2, $D$ and $E$ ). Each leaflet was suspended by 3 cords placed in a similar fashion (Figure 2, F-H). The pulmonary artery was then clamped. The newly reconstructed valve was tested with warm normal saline with the heart beating to recreate a normal anatomy of the distended right ventricle. Postoperative echocardiographic analysis demonstrated normal function of the reconstructed TV and subvalvular apparatus (Figure 1,B). The patient is asymptomatic at 3 months of follow-up yet continues to abuse drugs.

\section{Discussion}

Surgical treatment of acute right-sided endocarditis is associated with significant mortality. ${ }^{1}$ Compete destruction of the TV in a noncompliant patient is a difficult problem. These patients often leave the hospital despite medical advice once they feel better and do not complete the full course of antibiotics. Recurrences of endocarditis are common. Replacement of the valve with a prosthesis carries a very high risk of prosthetic endocarditis, whereas valve excision results in massive tricuspid regurgitation. Although successful repair of acute TV endocarditis involving a single leaflet has been reported, ${ }^{2}$ a complete reconstruction after total resection of the TV is a much more challenging procedure. A simplified technique for

* Gore-Tex cord, registered trademark of W. L. Gore \& Associates, Inc, Newark, Del. 

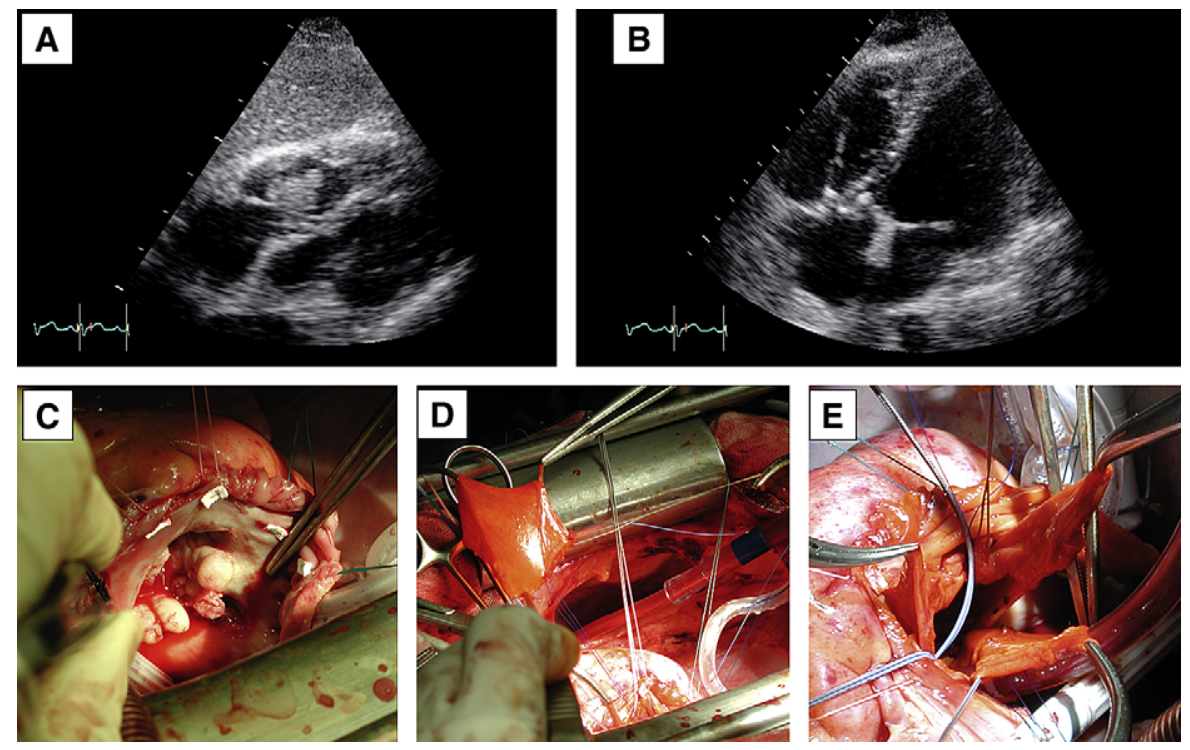

B

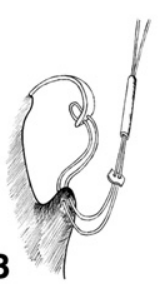

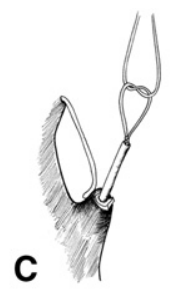
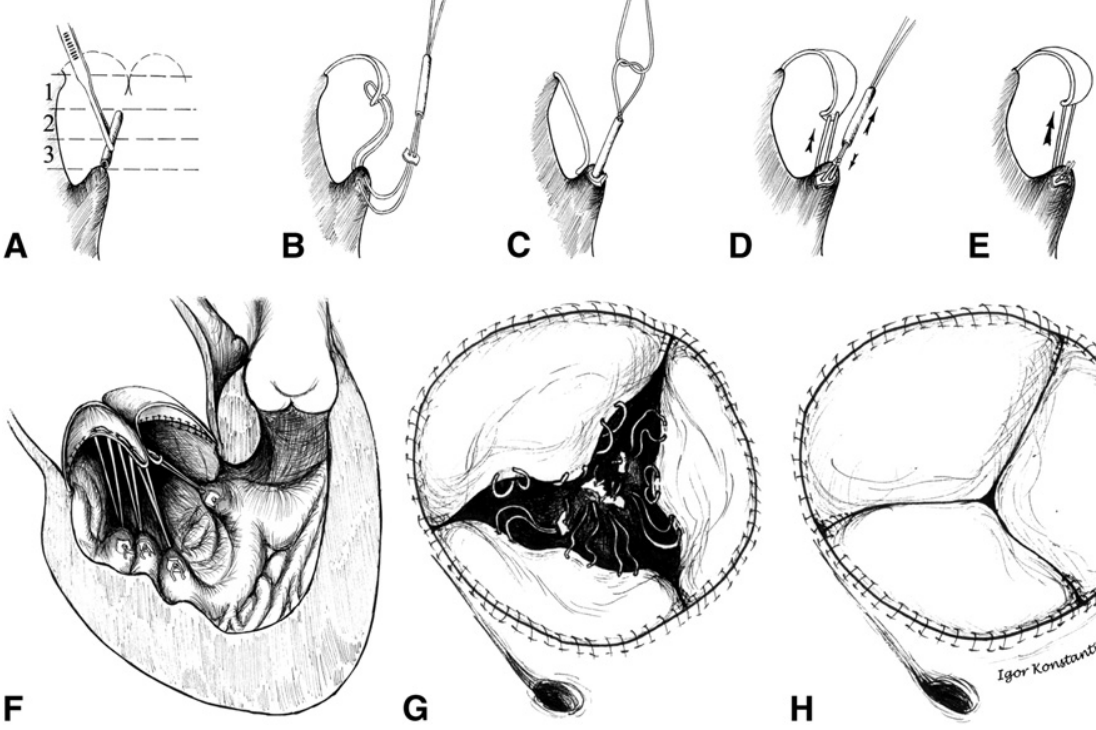

Figure 1. Echocardiogram demonstrating large vegetations on the tricuspid valve preoperatively $(A)$ and completely reconstructed tricuspid valve with artificial cords postoperatively (B). Intraoperative view showing tricuspid valve vegetations (C) and autologous pericardial leaflet reconstruction ( $D$ and $E$ ).
Figure 2. Schema illustrating a technique of complete reconstruction of the tricuspid valve. multiple cord placements for the entire cordal apparatus has been described for mitral valve repair. ${ }^{3,4}$ In reconstruction of the TV, however, one has to account for distension of the right ventricle during reconstruction of the $\mathrm{TV}$, especially in patients with dilated right ventricles. Thus polytetrafluoroethylene neocords need to be sized individually (Figure 2, $A-E$ ). This elegant technique of making individually measured artificial polytetrafluoroethylene cords was first described for mitral valve repair by Brizard and Carpentier. ${ }^{5}$ This technique was particularly useful during valvular reconstruction in our patient after total resection of the TV. The technique of complete valvular reconstruction described herein might be a valuable alternative in a noncompliant patient with an acute infective endocarditis.

I thank Mr Graham Jenkins and Dr Trenton Barrett for their help in preparing the illustrations.

\section{References}

1. Musci M, Siniawski H, Pasic M, et al. Surgical treatment of right-sided active infective endocarditis with or without involvement of the left heart: 20-year single center experience. Eur J Cardiothorac Surg. 2007;32: $118-25$.

2. Allen MD, Slachman F, Eddy AC, Cohen D, Otto CM, Pearlman AS. Tricuspid valve repair for tricuspid valve endocarditis: tricuspid valve "recycling." Ann Thorac Surg. 1991;51:593-8.

3. Konstantinov IE, Jalali H. Total chordal augmentation in a child with Marfan syndrome and severe mitral insufficiency. Tex Heart Inst J. 2007;34:423-4.

4. Tam R, Joshi P, Konstantinov IE. A simple method of preparing artificial chordae for mitral valve repair. J Thorac Cardiovasc Surg. 2006;132: 1486-7.

5. Brizard C, Carpentier A. Congenital malformation of the mitral valve. In: Stark JF, De Leval MR, Tsang VT, eds. Surgery for congenital heart defects, 3rd ed. Chichester, United Kingdom: John Wiley \& sons; 2006:573-90 